\title{
Aneurysmal subarachnoid haemorrhage: effect of CRHR1 genotype on fatigue and depression
}

\author{
Artur Vetkas ${ }^{1,2^{*}}$, Ele Prans ${ }^{2}$, Sulev Kõks ${ }^{3,4}$, Tõnu Rätsep ${ }^{1,2}$ and Toomas Asser ${ }^{1,2}$
}

\begin{abstract}
Background: Emotional health disturbances are common after aneurysmal subarachnoid hemorrhage (aSAH) and their causes are largely unexplored. Corticotropin-releasing hormone receptor 1 (CRHR1) is a key factor in stress reactivity and development of mental health disturbances after adverse life-events.

Methods: We explore the effect of CRHR1 genotype on mental health after aSAH in a retrospective cohort study. One hundred twenty-five patients have been assessed using EST-Q mental health questionnaire. Genotyping of CRHR1 single nucleotide polymorphisms (SNP-s) was performed (Rs7209436, Rs110402, Rs242924).

Results: Fatigue was present in almost half of aSAH patients, depression and anxiety in one-third. There was a high prevalence of insomnia and panic complaints. Rs 110402 minor allele decreased the risk of depression $(\mathrm{OR}=0.25$, $p=0.027$ for homozygotes). Depression was present in $14 \%$ vs $41 \%$ in minor and major allele homozygotes, respectively. Rs1 10402, Rs242924 and Rs7209436 minor alleles and TAT-haplotype, formed by them, were protective against fatigue. After Bonferroni correction only the association of Rs 110402 with fatigue remained statistically significant ( $O R=0.21, p=0.006$ for minor allele homozygotes). Results remained statistically significant when adjusted for gender, admission state, age and time from aSAH. In multiple regression analysis occurrence of fatigue was dependent on anxiety, modified Rankin score and Rs110402 genotype $\left(R^{2}=0.34, p<0.001\right)$.

Conclusions: CRHR1 minor genotype was associated with a lower risk of fatigue and depression after aSAH. Genetic predisposition to mental health disturbances associated with negative life-events could be a risk factor for fatigue and depression after aSAH and selected patients might benefit from advanced counselling in the recovery phase.
\end{abstract}

Keywords: Aneurysm, Subarachnoid haemorrhage, Fatigue, Depression, Mental health, Cortitrophin-releasing hormone receptor-type 1, CRHR1, Gene, SNP

\section{Background}

Incidence of aneurysmal subarachnoid haemorrhage $(\mathrm{aSAH})$ is around 7.9 per 100,000 patient years [1]. Up to $65 \%$ survive the subacute phase and two-thirds of those patients recover to functional independence $[2,3]$.

\footnotetext{
*Correspondence: Artur.Vetkas@gmail.com

${ }^{1}$ Tartu University Hospital, Tartu, Estonia

${ }^{2}$ Tartu University, Tartu, Estonia

Full list of author information is available at the end of the article
}

Only $15 \%$ develop a focal neurological deficit or need assistance in ambulation [4]. At the same time, many aSAH patients experience emotional and cognitive problems - anxiety and depression occurs in up to half of aSAH cases, post-traumatic stress disorder (PTSD) is reported in up to a third and occasional or constant fear of recurrent bleeding in two-thirds of patients $[5,6]$. Decrease in health-related quality of life is a welldocumented sequela of aSAH and changes in mental

C C The Author(s). 2020 Open Access This article is licensed under a Creative Commons Attribution 4.0 International License, which permits use, sharing, adaptation, distribution and reproduction in any medium or format, as long as you give appropriate credit to the original author(s) and the source, provide a link to the Creative Commons licence, and indicate if changes were made. The images or other third party material in this article are included in the article's Creative Commons licence, unless indicated otherwise in a credit line to the material. If material is not included in the article's Creative Commons licence and your intended use is not permitted by statutory regulation or exceeds the permitted use, you will need to obtain permission directly from the copyright holder. To view a copy of this licence, visit http://creativecommons.org/licenses/by/4.0/ The Creative Commons Public Domain Dedication waiver (http://creativecommons.org/publicdomain/zero/1.0/) applies to the data made available in this article, unless otherwise stated in a credit line to the data. 
component of quality of life were recently associated with CRHR1 genotype [3, 7].

The amount of emotional disturbances after aSAH seems to be out of proportion to cognitive and neurological impairments and causes of these changes are largely unexplored. Genetic predisposition might have a role in the formation of psychological disturbances after aSAH. The hypothalamic-pituitary-adrenal (HPA) axis response to stress is moderated by genetic and environmental factors and is implicated in pathogenesis of emotional diseases [8]. Genes that regulate the function of the stress response system are probable moderators of the effect that adverse life events have on the development of emotional illness [9].

Corticotropin-releasing hormone $(\mathrm{CRH})$ is a key stress mediator in the central nervous system [10]. Corticotropinreleasing hormone receptor 1 (CRHR1) is central in activating mesolimbic and HPA responses to different types of stress. CRHR1 genotype has been associated with mental health disorders and response to antidepressant treatment [11]. It has been reported to moderate the effects of stressful life events on development of major depression and moderate stress related cortisol reactivity $[12,13]$. Cortisol response to stress has been associated with depression and anxiety symptoms [14]. An elevated cortisol response is generally associated with major depression (MD) [15], and a flattened cortisol response is associated with panic disorder and post-traumatic stress disorder (PTSD) [16].

Few genetic studies have been conducted on the topic of neuropsychological disturbances after aSAH [16]. The objective of current research was to characterize specific neuropsychiatric disturbances after aSAH and explore the influence of CRHR1 genotype on their occurrence.

\section{Methods}

We performed a retrospective cohort study of long-term outcome of 125 surgically treated aSAH survivors. The studied group was selected from medical records of all patients diagnosed with aSAH $(n=467)$ from January 2001 to November 2013 in a university clinic (spontaneous ICH and other SAH causes were excluded). Selection criteria are presented in Fig. 1.

The average age at ictus was 54 years $(\mathrm{SD}=13$; range 24-82 years). The average time between initial hospitalisation and the study was 4 years $(\mathrm{SD}=2.8$; range $1-13$ years). $41 \%(n=51)$ of the patients were studied more than 3 years from ictus. $70 \%(n=88)$ of the patients were female. $78 \%(n=88)$ of the patients had more than 10 years of education. One patient was diagnosed with depression prior to aSAH based on the national database information available from 2009. The patients were interviewed in person with a structured questionnaire and blood samples were gathered. All participants gave
467 identified patients with aSAH (2001 - 2013)

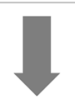

185 survivors with available contact information contacted by phone/letter

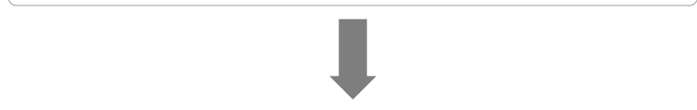

Exclusion criteria:

-31 could not be contacted

-19 declined to take part in the study or give blood

-9 unable to take part due to logistic reasons or severe comorbidities

-1 was excluded due to severe cognitive impairment

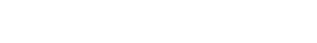

125 participants interviewed in person

Fig. 1 Selection of patients

their written informed consent and ethical approval was received from the institutional ethics committee.

\section{General management of patients}

All aSAH patients were managed according to general guidelines and our protocol is previously published [17]. All patients were admitted acutely and SAH was diagnosed by computer tomography (CT) or lumbar puncture. The aneurysm was assessed by CT-angiography or digital subtraction angiography. All patients were initially managed in a neurointensive care unit. Almost all patients were acutely operated upon, preferably via a pterional approach, and the aneurysms were clipped using standard microsurgical techniques. Endovascular procedures were preferentially performed in a separate institution during this time. Due to this our study includes a series of clipped patients. Clinical data of the patients and complications are presented in Table 1.

\section{Procedure}

Clinical data and Hunt and Hess grade $(\mathrm{HH})$ [18], which is a clinical grading system designed to predict prognosis and outcome in aSAH, were recorded at ictus. Remaining data was collected during the follow-up evaluation, when the patients were interviewed in person with a structured questionnaire (Additional file 1). Patient clinical recovery was evaluated according to the modified Rankin Scale (mRS) during the clinical interview [19]. Patients were also questioned about treatment for emotional disorders after aSAH, comorbidities, education and social living situation (living with family/ 
Table 1 Characteristics of aSAH, complications and clinical outcome

\begin{tabular}{|c|c|}
\hline Characteristic & N (\%) \\
\hline Male & $37(30 \%)$ \\
\hline Female & $88(70 \%)$ \\
\hline \multicolumn{2}{|l|}{ Hunt Hess score } \\
\hline 1 & $17(14 \%)$ \\
\hline 2 & $66(53 \%)$ \\
\hline 3 & $23(18 \%)$ \\
\hline 4 & $14(11 \%)$ \\
\hline 5 & $5(4 \%)$ \\
\hline \multicolumn{2}{|l|}{ Aneurysm location } \\
\hline ICA & $40(32 \%)$ \\
\hline AcomA & $44(35 \%)$ \\
\hline MCA & $22(18 \%)$ \\
\hline ACA & $8(6 \%)$ \\
\hline $\mathrm{BA}$ & $9(7 \%)$ \\
\hline VA & $2(2 \%)$ \\
\hline Intracerebral haemorrhage & $22(18 \%)$ \\
\hline Symptomatic vasospasm & $34(27 \%)$ \\
\hline Ischemic lesions on $C T$ & $25(20 \%)$ \\
\hline \multicolumn{2}{|l|}{ Hydrocephalus } \\
\hline acute & $43(34 \%)$ \\
\hline chronic & $14^{\mathrm{a}}(11 \%)$ \\
\hline \multicolumn{2}{|l|}{ Modified Rankin Score } \\
\hline 0 & $4(3 \%)$ \\
\hline 1 & $7(6 \%)$ \\
\hline 2 & $57(46 \%)$ \\
\hline 3 & 49 (39\%) \\
\hline 4 & $8(6 \%)$ \\
\hline
\end{tabular}

Abbreviations: ICA internal carotid artery, AcomA anterior communicating artery, MCA middle cerebral artery, ACA anterior cerebral artery, $B A$ basilar artery, VA vertebral artery, $C T$ computer tomography. ${ }^{a}$ These 14 patients required ventriculoperitoneal shunting after $\mathrm{aSAH}$

someone else or alone). Emotional State Questionnaire (EST-Q) was used to measure emotional health.

EST-Q is a well-validated self-rating scale that contains scales of Depression, Anxiety, Agoraphobia-Panic, Fatigue and Insomnia [20]. The items of EST-Q were derived from diagnostic criteria of DSMIV and ICD-10. Each item is rated by occurrence on a five-point scale ranging from 0 to 4 (respectively 'not' and 'all the time'). According to the results of factor analysis, EST-Q scales have demonstrated internal consistency of 0.69-0.88 Cronbach alpha. The participants were asked to report how much the various problems troubled them during the past 4 weeks. Scoring more than the cut-off point in a specific scale shows that the scale score is in the same magnitude as that of most patients with the given medical condition. The cut-off points for clinically important symptomatology were $\geq 12$ points for depression and anxiety, $\geq 8$ points for fatigue, $\geq 7$ points for agoraphobia-panic and $\geq 6$ points for insomnia [21]. EST-Q includes a question about frequency of 'Recurrent thoughts of death or suicide'. We have described the methodology of EST-Q in more detail previously [17]. The data was compared with an age and gender matched general population of 3923 subjects (obtained from the 6434 respondents of a National Health Interview Survey).

\section{Genotyping}

The genomic DNA was extracted from venous blood samples in $4 \mathrm{ml}$ EDTA containing vaccuettes by using the standard salting-out method. The EDTA tubes were stored at $-20 \mathrm{C}$ until DNA extraction. Isolated DNA was dissolved in Tris-EDTA (TE) buffer. The purity and concentrations of the DNA were measured by a spectrophotometer (NanoDrop, ND-1000). The gDNA samples were aliquotted and stored at $-80 \mathrm{C}$ until usage. Genotyping of marker single nucleotide polymorphisms (SNP) Rs7209436, Rs110402, Rs242924 and Rs242939 was carried out by using TaqMan SNP Genotyping Assay (Applied Biosystems, Foster City, CA, USA), which is a multiplex endpoint assay that detects variants of a single nucleic acid sequence. PCR reactions were run on the ViiA7 instrument (Applied Biosystems, Foster City, CA, USA) by using the following cycling parameters: after the first step at $95 \mathrm{C}$ for $10 \mathrm{~min}, 40$ cycles of denaturation at $92 \mathrm{C}$ for $15 \mathrm{~s}$ and extension at $60 \mathrm{C}$ for $1 \mathrm{~min}$. Genomic DNA (20 ng/ul) was amplified in a total volume of $10 \mathrm{ul}$ containing 1x Amplification Master Mix (Applied Biosystems, Foster City, CA, USA) and 1x probe. Genotypes were analysed by using the allelic discrimination function of the system (Table 2). There was no statistically significant difference between minor and major CRHR1 genotype with sociodemographic characteristics.

\section{Statistics}

Student's t-test was used to compare patients and age and gender matched general population EST-Q scores and determine the associations with clinical or sociodemographic factors. All continuous variables were controlled for normality using Shapiro-Wilk's W test. Logistic regression analysis was performed to study the association of CRHR1 genotype and EST-Q scores and calculate odds ratios (OR). EST-Q scores were used as indicator variables characterizing whether the individual possesses the emotional disturbances or not according to the cut-off values. In the SNP analysis we chose between additive/dominant/recessive model based on the AIC (Akaike information criterion) of the unadjusted model (recessive model - minor allele homozygote, 
Table 2 CRHR1 allele distribution $(n=125)$

\begin{tabular}{llllll}
\hline SNP & Genotype $(n)$ & & Minor allele $(n)$ & Major allele $(n)$ \\
\hline Rs7209436 & C/C (34) & C/T (69) & T/T (22) & T (91) & C (103) \\
Rs110402 & G/G (29) & A/G (68) & A/A (28) & A (96) & G (97) \\
Rs242924 & G/G (31) & G/T (67) & T/T (27) & $T(94)$ & G (98) \\
\hline
\end{tabular}

Abbreviations: CRHR1 corticotropin-releasing hormone receptor 1, SNPsingle nucleotide polymorphism

dominant model - major allele homozygote, additive model - OR for heterozygotes, which is multiplied in case of minor allele addition (minor homozygote - OR $\mathrm{x}$ OR)). When recessive genotype was rare (3 or less patients) then dominant model was preferred. Results were considered significant if $p<0.05$. The $p$-values that survived the Bonferroni correction are marked in bold. Pearson's correlation was used to asses internal correlation in EST-Q scales and logistic regression analysis was used to describe the influence of CRHR1 genotype (frequency of minor alleles) and patient factors on fatigue. Statistical analysis was performed with Stata 14.2 (StataCorp LLC) and SPSS 24 (IBM). Statistical analysis was performed with $\mathrm{R}$ (The $\mathrm{R}$ Foundation) and Stata 14.2 (StataCorp LLC).

\section{Results}

The average age at follow-up was 58 years $(\mathrm{SD}=12$, range $26-82) .78 \%(n=97)$ of the patients were living with family or someone else. $67 \%(n=84)$ had hypertension, $14 \%(n=18)$ joint pain or rheumatoid arthritis, $7 \%$ $(n=9)$ had diabetes and $2 \%(n=3)$ reported myocardial infarction. $24 \%(n=30)$ of the patients received metoprolol, $17 \%(n=21)$ amlodipine, $11 \%(n=14)$ ramipril, $8 \%(n=10)$ telmisartan, 6\% $(n=8)$ enalapril, and 6\% $(n=8)$ received perindopril/indapamide. Other medications were less common. $41 \%(n=51)$ of the patients required help in everyday life. $24 \%(n=30)$ of the patients saw a psychologist or psychiatrist and $38 \%(n=47)$ used antidepressants or similar medication at some point after aSAH.

Of the patients, $55 \%(n=68)$ had a mRS score of $0-2$ and $39 \%(n=48)$ had a score of 3 . Worse mRS score was associated with female gender $(2.5(\mathrm{SD}=0.8)$ vs 2.1 $(\mathrm{SD}=0.8), p=0.019)$ and requiring more daily help (3.1 $(\mathrm{SD}=0.5)$ vs $2.0(\mathrm{SD}=0.7), p<0.001)$.

\section{Emotional outcome scores}

Patients scored significantly worse on all EST-Q scales compared to the general population (Table 3). Female gender was associated with worse EST-Q scores: depression (mean $9(\mathrm{SD}=6.8)$ vs $5.6(\mathrm{SD}=4.7), p=0.006)$, anxiety (mean $9.2(\mathrm{SD}=5.8)$ vs $6.7(\mathrm{SD}=4.9), p=0.021)$, agoraphobia-panic (mean $3.8(\mathrm{SD}=4.3)$ vs $1.2(\mathrm{SD}=2)$, $p<0.001)$, fatigue (mean $7.2(\mathrm{SD}=4.2)$ vs $5.4(\mathrm{SD}=4)$, $p=0.03)$, and insomnia (mean $5.1(\mathrm{SD}=3.3)$ vs $3.7(\mathrm{SD}=$ 3.6), $p=0.04)$. A worse fatigue score was associated with having hypertension (mean 7.5 ( $\mathrm{SD} \pm 4.2$ ) vs 4.9 ( $\mathrm{SD} \pm$ 3.6), $p<0.001$ ) and having joint pains (mean 8.6 ( $\mathrm{SD} \pm$ $3.4)$ vs 6.4 ( $\mathrm{SD} \pm 4.3), p=0.021)$. Taking amlodipine was associated with a worse depression score (10.7 (SD \pm 6.9$)$ vs mean 7.5 ( $\mathrm{SD} \pm 6.3$ ), $p=0.035$ ).

aSAH patients had a high prevalence of those scoring above the cut-off values for emotional disturbances compared to the general population, with fatigue and insomnia being the most common problems presenting in around half of the patients (Fig. 2). About one third of the patients had higher than cut-off scores demonstrating depression $(29 \%, n=36)$ and anxiety $(30 \%, n=38)$. $15 \%$ of patients $(n=19)$ had higher than cut-off values on agoraphobia-panic scale. $14 \%(n=18)$ of aSAH patients had frequent thoughts of death or suicide (ranging from sometimes to constant) compared to $3 \%(n=126)$ in the general population $(p<0.001)$.

\section{Association of genotype with EST-Q outcomes}

In regression analysis we explored the association of CRHR1 genotype with emotional disorder symptoms (Table 4). The results remained significant after adjustment for gender, neurological state at admission $(\mathrm{HH})$, patient age and time from aSAH to evaluation. Effect of Rs110402 on fatigue remained statistically significant after Bonferroni correction for multiple comparisons.

Table 3 EST-Q scores among aSAH patients $(n=125)$ and age and gender matched general population $(n=3923)$

\begin{tabular}{llllll}
\hline EST-Q scales & aSAH mean, $n=125$ & aSAH SD & Population mean, $n=3923$ & Population SD & $p$-value \\
\hline Depression & 8 & 6.5 & 4.3 & 5.4 & $<.4$ \\
Anxiety & 8.5 & 5.6 & 4.5 & 0.001 \\
Agoraphobia-Panic & 3 & 3.9 & 0.9 & 2.6 & $<0.001$ \\
Fatigue & 6.7 & 4.2 & 3.1 & 2.7 & $<.001$ \\
Insomnia & 4.7 & 3.4 & 3.2 & 3 & $<0.001$ \\
\hline
\end{tabular}

Abbreviations: $S D$ standard deviation, $p$-value 


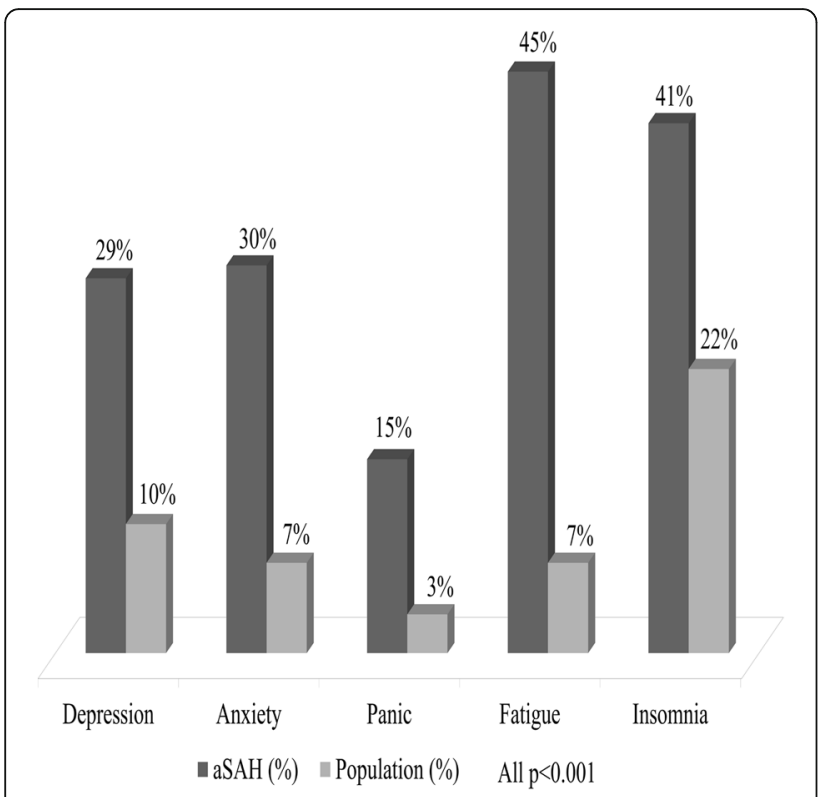

Fig. 2 Prevalence (\%) of emotional disorders according to cut-off values among aSAH patients $(n=125)$ and age and gender matched general population ( $n=3923$ ) based on the EST-Q cut-off scores (all $p<0.001$ )

Rs110402 minor genotype carriers had a reduced risk of depression (OR $=0.5,95 \% \mathrm{CI}, 0.28-0.92, p=0.027)$ in the additive model. Among patients with the minor Rs110402 alleles prevalence of depression was close to the general population (14\%) and much lower than in patients with the major genotype (41\%) (Fig. 3).

CRHR1 minor alleles (Rs7209436, Rs110402, Rs242924) had a protective effect against developing fatigue $(\mathrm{OR}=0.22-0.46, p<0.05)$ in additive and recessive models. Prevalence of fatigue was $62 \%$ vs $25 \%$ in major and minor allele homozygotes of Rs110402, respectively (Fig. 3). TAT haplotype, formed by the three minor alleles, was associated with a significant protective effect against fatigue $(\mathrm{OR}=0.26,95 \% \mathrm{CI}, 0.08-0.81, p=0.021)$ in the additive model.
Rs242939 minor allele had a protective effect against insomnia when adjusted for gender, Hunt Hess score, patient age and time of evaluation from aSAH $\left(\mathrm{OR}^{*}=\right.$ $\left.0.43,95 \% \mathrm{CI}, 0.18-1.02, \mathrm{p}^{*}=0.057\right)$ in the additive model (Table 4).

\section{Internal correlation and factors influencing fatigue}

Almost half of aSAH patients had higher than cut-off EST-Q scores indicating fatigue $(45 \% ; n=56)$ and insomnia $(41 \% ; n=51)$. Mental health disorders, as diagnosed by EST-Q cut-off values, were significantly correlated with each other (Table 5). There was a moderate correlation between mRS and EST-Q scales, with higher values for fatigue and depression. Rs110402 was significantly correlated to fatigue and depression and Rs7209436 to fatigue.

We performed a multiple regression analysis for fatigue due to its reported association with anxiety and depression. The best model for fatigue included anxiety, mRS and Rs110402 genotype (number of minor alleles) with a $\mathrm{R}^{2}=0.34, p<0.001$ (Table 6). In a separate analysis, with insomnia entered instead of mRS, the model still had good explanatory value, but $R^{2}$ decreased to $0.31, p<0.001$. Sociodemographic factors and comorbidities were not associated with fatigue in multiple regression analysis.

\section{Discussion}

We describe an association of CRHR1 genotype with mental health after aSAH. Multiple logistic regression analysis showed that corticotropin-releasing hormone receptor 1 genotype (Rs7209436, Rs110402, Rs242924) was associated with mental health disorders measured with EST-Q. Rs110402 minor allele decreased the risk of depression ( $\mathrm{OR}=0.25, p=0.027$ for minor allele homozygotes). Rs110402, Rs242924 and Rs7209436 minor alleles and TAT-haplotype formed by them were protective against developing fatigue $(\mathrm{OR}=0.22-0.46$, $p<0.05)$. Only association of Rs110402 with fatigue

Table 4 Influence of genotype on EST-Q outcomes (only statistically significant results are reported)

\begin{tabular}{|c|c|c|c|c|c|c|c|c|}
\hline SNP & Allele & Model & OR & $95 \% \mathrm{Cl}$ & $p$ & $O R^{*}$ & $95 \% \mathrm{Cl}^{*}$ & $p^{*}$ \\
\hline \multicolumn{9}{|l|}{ Depression } \\
\hline Rs110402 & Minor & Additive & 0.50 & $0.28-0.92$ & 0.027 & 0.50 & $0.26-0.94$ & 0.032 \\
\hline \multicolumn{9}{|l|}{ Fatigue } \\
\hline Rs110402 & Minor & Additive & 0.46 & $0.26-0.8$ & 0.006 & 0.48 & $0.27-0.85$ & 0.012 \\
\hline Rs7209436 & Minor & Recessive & 0.22 & $0.07-0.69$ & 0.009 & 0.23 & $0.07-0.75$ & 0.015 \\
\hline Rs242924 & Minor & Recessive & 0.35 & $0.14-0.9$ & 0.030 & 0.37 & $0.14-0.98$ & 0.044 \\
\hline TAT-haplotype & Minor & Additive & 0.26 & $0.08-0.81$ & 0.021 & 0.27 & $0.08-0.88$ & 0.030 \\
\hline \multicolumn{9}{|l|}{ Insomnia } \\
\hline Rs242939 & Minor & Additive & 0.43 & $0.18-1.02$ & 0.057 & 0.38 & $0.15-0.96$ & 0.042 \\
\hline
\end{tabular}

Abbreviations: $\mathrm{OR}$ odds ratio, $\mathrm{Cl}$ confidence interval. $P$-values that are adjusted for gender, Hunt Hess score, patient age and time of evaluation from aSAH are marked with *. P-values that survived the Bonferroni correction are marked with bold 


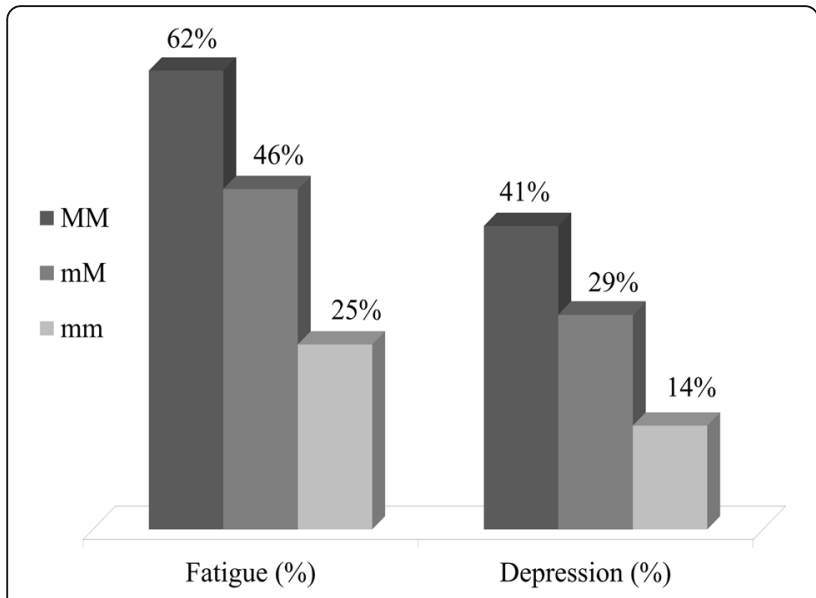

Fig. 3 Prevalence of fatigue and depression based on cut-off values in aSAH patients ( $n=125)$ according to Rs 110402 to genotype. MM - homozygote for major allele, mM - heterozygote, mm homozygote for minor allele

remained statistically significant after Bonferroni correction ( $\mathrm{OR}=0.21, p=0.006$ for minor allele homozygotes). Rs242939 minor genotype was associated with a lower prevalence of insomnia in the adjusted model.

Our study shows that aSAH patients frequently experience long-term mental health disturbances. Emotional disturbances have been reported after stroke. A recent study described similarities among patient perceived outcomes in quality of life components after different types of stroke (ischemic stroke, intracerebral hematoma, and SAH) [22]. These patients, similar to aSAH, experience a severe life-changing event, which requires habituation and acquiring a new social role. Poststroke depression occurs in up to $33 \%$ and anxiety in up to $25 \%$ of cases, with reported associations between them $[23,24]$. In our study almost $1 / 3$ of aSAH patients scored above the cut-off values of EST-Q indicating depression and anxiety. $20 \%(n=25)$ showed a coexistence of the two conditions. Depression and anxiety were moderately correlated with each other (Pearson's $r=$
$0.54, p<0.001)$ and presented more often among women.

Almost half of the patients in our study (45\%) scored above the cut-off value for fatigue. Fatigue is considered to be the most common complaint after stroke and it occurs in up to $50 \%$ of cases [25]. Fatigue could be defined as "a state characterized by weariness unrelated to previous exertion levels and usually not ameliorated by rest" [26]. Fatigue is considered to be a separate entity from other mental health disorders and its occurrence after neurological diseases is more frequent than would be expected based on age and disability [27]. Among younger patients with stroke, fatigue predicts a worse health-related quality of life than neurologic dysfunction [28]. Fatigue has a detrimental effect on quality of life by restricting everyday activities and it can persist due to a state of mental exhaustion from having to deal with processes of rehabilitation and adaptation. Poststroke fatigue has been associated with anxiety and depression, but it can occur separately from them [29]. Fatigue can persist after successful treatment of major depression and it requires distinct interventions [30]. In our study fatigue showed a moderate correlation to anxiety (Pearson's $r=0.49, p<0.001)$ and depression $(r=0.42, p<$ 0.001 ). In a multiple regression model, $34 \%$ of variance in fatigue was best explained by anxiety, mRS score and Rs110402 genotype, $p<0.001)$. This implies that fatigue is related both to physical and mental complaints of the patients and requires a multidisciplinary management.

We describe a significant number of patients reporting thoughts of death and suicide $(14 \%, n=18)$, which reflects on the extent of their emotional problem. Suicidality was not associated with CRHR1 genotype in our study. Patients also had a high prevalence of agoraphobia-panic complaints and twice as much sleep problems as the general population. $70 \%$ of the patients in our study were female and female gender was associated with worse EST-Q scores.

The neural events of mental health disturbances after aSAH, measured in this study with EST-Q, remain largely unknown. Hormonal changes have been proposed as a cause, but studies reported varying results

Table 5 Correlations between EST-Q scales and CRHR1 genotype in aSAH patients $(n=125) .{ }^{*} p<0.001 ;{ }^{* *} p<0.01 ;{ }^{* * *} p<0.05$

\begin{tabular}{|c|c|c|c|c|c|c|c|c|}
\hline Variables & Fatigue & Depression & Anxiety & Panic-Agorophobia & Insomnia & $\mathrm{mRS}$ & Rs110402 & Rs7209436 \\
\hline Depression & $0.42^{*}$ & & & & & & & \\
\hline Anxiety & $0.49^{*}$ & $0.54^{*}$ & & & & & & \\
\hline Panic-Agorophobia & $0.34^{*}$ & $0.47^{*}$ & $0.59^{*}$ & & & & & \\
\hline Insomnia & $0.33^{*}$ & $0.33^{*}$ & $0.34^{*}$ & $0.28^{* *}$ & & & & \\
\hline $\mathrm{mRS}$ & $0.4^{* *}$ & $0.34^{* *}$ & $0.29^{* *}$ & $0.28^{* *}$ & $0.23^{* *}$ & & & \\
\hline Rs110402 & $-0.25^{* * *}$ & $-0.20^{* * *}$ & -0.12 & -0.06 & -0.06 & -0.14 & & \\
\hline Rs7209436 & $-0.23^{* * *}$ & -0.15 & -0.12 & -0.06 & -0.1 & -0.14 & $0.89^{* * *}$ & \\
\hline Rs242924 & -0.15 & -0.15 & -0.07 & -0.01 & -0.06 & -0.16 & $0.86^{* * *}$ & $0.9^{* * *}$ \\
\hline
\end{tabular}


Table 6 Multiple regression for fatigue occurrence based on cut-off values

\begin{tabular}{lllll}
\hline Variables & B & SE & $\beta$ & $\boldsymbol{p}$-value \\
\hline Anxiety & 0.42 & 0.08 & 0.39 & $<0.001$ \\
mRS & 0.016 & 0.05 & 0.27 & 0.001 \\
Rs110402 & -0.12 & 0.06 & -0.17 & 0.027 \\
\hline
\end{tabular}

[31]. Coping strategies and premorbid psychiatric history could affect the long-term outcome of patients [32], but are hard to study retrospectively. It is possible that patients have a psychological reaction to a sudden traumatic event and live with a recurrent fear of rebleeding that affects their emotional well-being [6]. Patients might be stigmatised by the disease or there is a distinct neurobiological predisposition for psychological maladaptation after aSAH. Understanding that a person with an intrinsic vulnerability is at more risk of developing a mental illness or an inadequate psychological reaction to a stressful life event is well acknowledged in the psychiatric community [33].

It is unknown whether the studied SNP-s are functional or they are in linkage disequilibrium with other regions, but it has been previously reported that CRHR1 genotype is related to formation of major depression after stressful life events and could moderate cortisol reactivity to stress $[12,13]$. In a multilocus genetic profile score study, CRHR1 together with other HPA genes has been shown to interact with chronic stress moderating diurnal cortisol slope and predicting fatigue [34]. CRHR1 genotype has been associated with PTSD and depression symptoms in survivors of post-surgical intensive care unit treatment [35]. Cortisol dysbalance occurs in critical illness patients, with a possible insufficiency after aSAH [36]. Corticosteroid administration was associated with a decreased frequency of post intensive care PTSD symptoms after major surgery [37].

Emotional health disturbances after aSAH are reported rather unhomogenously. Some studies describe the patients as being depressed, having anxiety, chronically fatigued or experiencing PTSD symptoms. Psychiatric disturbances have a certain overlapping and a more uniform diagnostic strategy is required. There are few genetic studies related to outcomes after aSAH and their results have been inconclusive. One of the shortcomings is that outcomes were mostly measured with general scoring systems (Glasgow Outcome Scale, modified Rankin Scale), which are clinically relevant, but do not reflect on the entire state of patients recovery [5]. Health related quality of life was assessed separately in the same group of aneurysmal SAH patients using SF-36 questionnaire and it was reduced in all domains including mental health, where it was positively associated with minor CRHR1 genotype [7]. The strength of the current study is that patients were characterised using a sensitive assessment tool that showed an array of psychological symptoms with high prevalence in post-aSAH patients, including suicidal thoughts. To the best of our knowledge, this is the first study reporting a genetic association with specific mental health disturbances after aSAH. The different emotional problems that aSAH patients experience could be interconnected, but they most likely require distinct neurocognitive interventions and counselling. Mental health disturbances could interfere with patients returning to work and increase the disease burden [38, 39]. We describe a neurobiological predisposition for development of depression and fatigue after aSAH which could help in selecting patients at risk and potentially guiding their therapy.

\section{Limitation of the study}

Our study was a retrospective design and included 125 patients, due to this our results in SNP analysis might be underpowered to draw certain conclusions. Despite mental health diagnosis being extractable from the national database, we don't know the full extent of previous emotional problems in our patient group. Cultural factors could also influence outcomes. We lack information regarding the cognitive profile of the patients, but none of them had severe disabilities when interviewed. Our findings need to be validated in a larger prospective cohort and further studies are required to determine the functional consequences of CRHR1 genotype variability.

\section{Conclusions}

Results of our study suggest that prevalence of long-term emotional health disorders after aSAH is high with depression and anxiety presenting in one-third of patients. Fatigue is the most common problem and it is influenced both by mental and physical state of the patients. CRHR1 minor genotype was associated with less fatigue and depression symptoms after aSAH. The association with CRHR1 could be due to its role in the HPA-system response to stress and predisposition of patients to emotional problems after adverse life-events. New and improved biomarkers are needed to predict, diagnose and treat the long-term consequences of aSAH. Such biomarkers could help identify patients who would benefit from early neuropsychological rehabilitation and decrease the disease burden.

\section{Supplementary information}

Supplementary information accompanies this paper at https://doi.org/10. 1186/s12883-020-01727-y.

Additional file 1. The file contains a description of the EST-Q questionnaire used to asses mental health disturbances and general questions used to asses the recovery process of patients.

\section{Abbreviations}

aSAH: Aneurysmal subarachnoid hemorrhage; CRHR1: Corticotropin-releasing hormone receptor; SNP: Single nucleotide polymorphism; PTSD: Post- 
traumatic stress disorder; HPA axis: Hypothalamic-pituitary-adrenal axis; MD: Major depression; SF-36: Short Form 36; CT: Computer tomography; mRS: Modified Rankin Scale; EST-Q: Emotional State Questionnaire; HH: Hunt and Hess grade; DSMIV: Diagnostic and Statistical Manual of Mental Disorders 4th Edition; ICD-10: International Classification of Diseases 10th Edition; DNA: Deoxyribonucleic acid; EDTA: Ethylenediaminetetraacetic acid; TE: Tris-EDTA; AIC: Akaike information criterion; ICA: Internal carotid artery; AcomA: Anterior communicating artery; MCA: Middle cerebral artery; ACA: Anterior cerebral artery; BA: Basilar artery; VA: Vertebral artery; SD: Standard deviation; p: $p$-value; OR: Odds ratio; Cl: Confidence interval

\section{Acknowledgements}

None.

\section{Authors' contributions}

A.V. has contributed to organisation, conception and design of the study, has drafted the manuscript, prepared the tables and figures. E.P. Has performed the PCR analysis and participated in writing the genetic analysis section. S.K. Has contributed in organisation and genetic analysis section. T.R. has contributed to conception and design of the study. T.A. has contributed to organisation, conception and design of the study. All authors have read and approved the manuscript.

\section{Funding}

No funding was received for this research.

Three of the authors (EP, SK, TA) received grants from the Estonian Ministry of Education and Research. Other authors (AV, TR) have no financial disclosure. - Ele Prans, Sulev Kõks: This work was supported by institutional research grants IUT20-46 of the Estonian Ministry of Education and Research. - Toomas Asser: This work was supported by institutional research grants IUT2-4 of the Estonian Ministry of Education and Research.

\section{Availability of data and materials}

The datasets generated and/or analysed during the current study are not publicly available due to restrictions.

\section{Ethics approval and consent to participate}

All procedures performed in studies involving human participants were in accordance with the ethical standards of the institutional and/or national research committee and with the 1964 Helsinki declaration and its later amendments or comparable ethical standards.

Ethical approval (214/T-2/2012) was given by Tartu University ethical committee. Written informed consent was obtained from all individual participants included in the study. In case patients were not able to perform the written consent themselves legal guardian or representative provided consent to participate in their stead.

\section{Consent for publication}

Not applicable.

\section{Competing interests}

None. All authors certify that they have no affiliations with or involvement in any organization or entity with any financial interest (such as honoraria; educational grants; participation in speakers' bureaus; membership, employment, consultancies, stock ownership, or other equity interest; and expert testimony or patent-licensing arrangements), or non-financial interest (such as personal or professional relationships, affiliations, knowledge or beliefs) in the subject matter or materials discussed in this manuscript.

\section{Author details}

${ }^{1}$ Tartu University Hospital, Tartu, Estonia. ${ }^{2}$ Tartu University, Tartu, Estonia.

${ }^{3}$ Centre for Molecular Medicine and Innovative Therapeutics, Murdoch University, Perth, WA, Australia. ${ }^{4}$ The Perron Institute for Neurological and Translational Science, Perth, WA, Australia.

\section{Received: 31 July 2019 Accepted: 14 April 2020}

Published online: 18 April 2020

\section{References}

1. Etminan N, Chang H-S, Hackenberg K, de Rooij NK, Vergouwen MDI, Rinkel GJE, et al. Worldwide incidence of aneurysmal subarachnoid hemorrhage according to region, time period, blood pressure, and smoking prevalence in the population. JAMA Neurol. 2019;76:588-97. https:/doi.org/10.1001/jamaneurol.2019.0006.
2. Nieuwkamp DJ, Setz LE, Algra A, Linn FH, de Rooij NK, Rinkel GJ. Changes in case fatality of aneurysmal subarachnoid haemorrhage over time, according to age, sex, and region: a meta-analysis. Lancet Neurol. 2009;8:635-42. https://doi.org/10.1016/S1474-4422(09)70126-7.

3. Noble AJ, Schenk T. Which variables help explain the poor health-related quality of life after subarachnoid hemorrhage? A meta-analysis. Neurosurgery. 2010;66:772-83.

4. Mayer SA, Kreiter KT, Copeland D, Bernardini GL, Bates JE, Peery S, et al. Global and domain-specific cognitive impairment and outcome after subarachnoid hemorrhage. Neurology. 2002;45:875-82. https://doi.org/10. 1212/01.wnl.0000035748.91128.c2.

5. Rinkel GJE, Algra A. Long-term outcomes of patients with aneurysmal subarachnoid haemorrhage. Lancet Neurol. 2011;10:349-56. https://doi.org/ 10.1016/S1474-4422(11)70017-5.

6. Hütter B-O, Kreitschmann-Andermahr I. Subarachnoid hemorrhage as a psychological trauma. J Neurosurg. 2014;120:923-30.

7. Vetkas A, Prans E, Köks S, Rätsep T, Asser T. Aneurysmal subarachnoid haemorrhage: effect of CRHR1 genotype on mental health-related quality of life. Sci Rep. 2020;10:1-8.

8. Gerritsen L, Milaneschi Y, Vinkers $\mathrm{CH}$, van Hemert AM, van Velzen L, Schmaal L, et al. HPA Axis genes, and their interaction with childhood maltreatment, are related to cortisol levels and stress-related phenotypes. Neuropsychopharmacology. 2017;42: 2446-55. https://doi.org/10.1038/npp.2017.118.

9. Wasserman D, Wasserman J, Sokolowski M. Genetics of HPA-axis, depression and suicidality. Eur Psychiatry. 2010;25:278-80. https//doi.org/10.1016/j.eurpsy.2009.12.016.

10. Naughton M, Dinan TG, Scott LV. Corticotropin-releasing hormone and the hypothalamic-pituitary-adrenal axis in psychiatric disease. Handb Clin Neurol. 2014;124:69-91. https://doi.org/10.1016/B978-0-444-59602-4.00005-8.

11. Liu Z, Zhu F, Wang G, Xiao Z, Tang J, Liu W, et al. Association study of corticotropinreleasing hormone receptor 1 gene polymorphisms and antidepressant response in major depressive disorders. Neurosci Lett. 2007;414:155-8.

12. Liu Z, Liu W, Yao L, Yang C, Xiao L, Wan Q, et al. Negative life events and corticotropin-releasing-hormone receptor1 gene in recurrent major depressive disorder. Sci Rep. 2013;3:1-5. https://doi.org/10.1038/srep01548.

13. Sumner JA, McLaughlin KA, Walsh K, Sheridan MA, Koenen KC. CRHR1 genotype and history of maltreatment predict cortisol reactivity to stress in adolescents. Psychoneuroendocrinology. 2014;43:71-80. https:/doi.org/10.1016/j.psyneuen.2014.02.002.

14. Fiksdal A, Hanlin L, Kuras Y, Gianferante D, Chen X, Thoma MV, et al. Associations between symptoms of depression and anxiety and cortisol responses to and recovery from acute stress. Psychoneuroendocrinology. 2019;102:44-52. https://doi.org/10.1016/j.psyneuen.2018.11.035.

15. Chopra KK, Ravindran A, Kennedy SH, Mackenzie B, Matthews S, Anisman H, et al. Sex differences in hormonal responses to a social stressor in chronic major depression. Psychoneuroendocrinology. 2009;34:1235-41. https://doi. org/10.1016/j.psyneuen.2009.03.014.

16. McFarlane AC, Barton CA, Yehuda R, Wittert G. Cortisol response to acute trauma and risk of posttraumatic stress disorder. Psychoneuroendocrinology. 2011;36:720-7. https://doi.org/10.1016/j.psyneuen.2010.10.007.

17. Vetkas A, Lepik T, Eilat T, Rätsep T, Asser T. Emotional health and quality of life after aneurysmal subarachnoid hemorrhage. Acta Neurochir. 2013;155:1107-14.

18. Hunt WE, Hess RM. Surgical risk as related to time of intervention in the repair of intracranial aneurysms. J Neurosurg. 1968;28:14-20. https://doi.org/ 10.3171/jns.1968.28.1.0014.

19. Van-Swieten JC, Koudstaal PJ, Visser MC, Schouten HJ, van Gijn J. Interobserver agreement for the assessment of handicap in stroke patients. Stroke. 1988;19:604-7.

20. Aluoja A, Shlik J, Vasar V, Luuk K, Leinsalu M. Development and psychometric properties of the emotional state questionnaire, a self-report questionnaire for depression and anxiety. Nord J Psychiatry. 1999;53:443-9. https://doi.org/10.1080/080394899427692.

21. Oopik P, Aluoja A, Kalda R, Maaroos H-I. Screening for depression in primary care. Fam Pract. 2006;23:693-8. https://doi.org/10.1093/fampra/cml052.

22. Katzan IL, Schuster A, Newey C, Uchino K, Lapin B. Patient-reported outcomes across cerebrovascular event types. Neurology. 2018;91:e2182-91. https://doi.org/10.1212/WNL.0000000000006626.

23. Towfighi A, Ovbiagele B, El Husseini N, Hackett ML, Jorge RE, Kissela BM, et al. Poststroke depression: a scientific statement for healthcare professionals from the American Heart Association/American Stroke Association. Stroke. 2017;48: e30-43. https://doi.org/10.1161/STR.0000000000000113.

24. Wright F, Wu S, Chun H-YY, Mead G. Factors associated with Poststroke anxiety: a systematic review and meta-analysis. Stroke Res Treat. 2017;2017: 1-7. https://doi.org/10.1155/2017/2124743. 
25. Cumming TB, Packer M, Kramer SF, English C. The prevalence of fatigue after stroke: a systematic review and meta-analysis. Int J Stroke. 2016;11: 968-77. https://doi.org/10.1177/1747493016669861.

26. De Groot MH, Phillips SJ, Eskes GA. Fatique associated with stroke and other neurologic conditions: implications for stroke rehabilitation. Arch Phys Med Rehabil. 2003:84:1714-20

27. Kluger BM, Krupp LB, Enoka RM. Fatigue and fatigability in neurologic illnesses: proposal for a unified taxonomy. Neurology. 2013;80:409-16. https://doi.org/10.1212/WNL.0b013e31827f07be.

28. Becker K, Kohen R, Lee R, Tanzi P, Zierath D, Cain K, et al. Poststroke fatigue: hints to a biological mechanism. J Stroke Cerebrovasc Dis. 2015;24:618-21. https://doi.org/10.1016/j.jstrokecerebrovasdis.2014.10.008.

29. Ponchel A, Bombois $\mathrm{S}$, Bordet $\mathrm{R}$, Hénon $\mathrm{H}$. Factors associated with Poststroke fatigue: a systematic review. Stroke Res Treat. 2015;2015. https:// doi.org/10.1155/2015/347920.

30. Nierenberg AA, Keefe BR, Leslie VC, Alpert JE, Pava JA, Worthington JJ, et al. Residual symptoms in depressed patients who respond acutely to fluoxetine. J Clin Psychiatry. 1999;60:221-5. https://doi.org/10.4088/JCP v60n0403.

31. Booij HA, Gaykema WDC, Kuijpers KAJ, Pouwels MJM, den Hertog HM. Pituitary dysfunction and association with fatigue in stroke and other acute brain injury. Endocr Connect. 2018;7:R223-37. https://doi.org/10.1530/EC-180147.

32. Hedlund M, Zetterling M, Ronne-Engström E, Carlsson M, Ekselius L. Depression and post-traumatic stress disorder after aneurysmal subarachnoid haemorrhage in relation to lifetime psychiatric morbidity. Br J Neurosurg. 2011;25:693-700.

33. Goh C, Agius M. The stress-vulnerability model how does stress impact on mental illness at the level of the brain and what are the consequences? Psychiatr Danub. 2010;22:198-202.

34. Starr LR, Dienes K, Li YI, Shaw ZA. Chronic stress exposure, diurnal cortisol slope, and implications for mood and fatigue: moderation by multilocus HPA-Axis genetic variation. Psychoneuroendocrinology. 2019;100:156-63. https://doi.org/10.1016/j.psyneuen.2018.10.003.

35. Davydow DS, Kohen R, Hough CL, Tracy JH, Zatzick D, Katon WJ. A pilot investigation of the association of genetic polymorphisms regulating corticotrophin-releasing hormone with posttraumatic stress and depressive symptoms in medical-surgical intensive care unit survivors. J Crit Care. 2014; 29:101-6.

36. Lanterna LA, Spreafico V, Gritti P, Prodam F, Signorelli A, Biroli F, et al. Hypocortisolism in noncomatose patients during the acute phase of subarachnoid hemorrhage. J Stroke Cerebrovasc Dis. 2013;22:e189-96. https://doi.org/10.1016/j.jstrokecerebrovasdis.2012.11.002.

37. Schelling G, Roozendaal B, Krauseneck T, Schmoelz M, DE Quervain D, Briegel J. Efficacy of hydrocortisone in preventing posttraumatic stress disorder following critical illness and major surgery. Ann N Y Acad Sci. 2006; 1071:46-53. https://doi.org/10.1196/annals.1364.005.

38. Al-Yassin A, Ouyang B, Temes R. Depression and anxiety following aneurysmal subarachnoid hemorrhage are associated with higher six-month unemployment rates. J Neuropsychiatry Clin Neurosci. 2017;29:67-9. https:// doi.org/10.1176/appi.neuropsych.15070171.

39. Rivero-Arias O, Gray A, Wolstenholme J. Burden of disease and costs of aneurysmal subarachnoid haemorrhage (aSAH) in the United Kingdom. Cost Eff Resour Alloc. 2010;8:6. https://doi.org/10.1186/1478-7547-8-6.

\section{Publisher's Note}

Springer Nature remains neutral with regard to jurisdictional claims in published maps and institutional affiliations.

Ready to submit your research? Choose BMC and benefit from:

- fast, convenient online submission

- thorough peer review by experienced researchers in your field

- rapid publication on acceptance

- support for research data, including large and complex data types

- gold Open Access which fosters wider collaboration and increased citations

- maximum visibility for your research: over $100 \mathrm{M}$ website views per year

At $\mathrm{BMC}$, research is always in progress.

Learn more biomedcentral.com/submissions 